\title{
Individual Differences in Temporal Summation of Pain Reflect Pronociceptive and Antinociceptive Brain Structure and Function
}

\author{
Joshua C. Cheng, ${ }^{1,2}$ Nathalie Erpelding, ${ }^{1}$ Aaron Kucyi, ${ }^{1,2}$ Danielle D. DeSouza, ${ }^{1,2}$ and Karen D. Davis ${ }^{1,2,3}$ \\ ${ }^{1}$ Division of Brain, Imaging \& Behaviour Systems, Toronto Western Research Institute, Toronto M5T 2S8, Canada, ${ }^{2}$ Institute of Medical Science, University \\ of Toronto, Toronto M5S 2J7, Canada, and 32Department of Surgery, University of Toronto, Toronto M5T 1P5, Canada
}

\begin{abstract}
Temporal summation of pain (TSP), the perception of increasingly greater pain evoked by repetitive noxious stimuli, is highly variable between individuals. Individuals with facilitated pain processing and/or reduced pain-modulatory capabilities are regarded as pronociceptive, whereas individuals with reduced pain processing capacity are characterized as antinociceptive. Brodmann area (BA) 3a of the primary somatosensory cortex is part of an ascending pathway from the sensory thalamus that mediates TSP. Descending pain modulation involves projections from the subgenual anterior cingulate cortex (sgACC) to the periaqueductal gray to the rostral ventromedial medulla (RVM). Here, we tested the hypothesis that pronociceptive individuals have an enhanced TSP response compared with antinociceptive individuals, marked by facilitated ascending nociceptive processing and/or reduced capacity for descending pain modulation. Eighty healthy humans were tested with a TSP protocol and underwent structural and resting-state functional magnetic resonance imaging. We found large interindividual differences in TSP responses, which were positively correlated with functional connectivity (FC) between individuals' right sensory thalamus with their BA 3a (thal-BA 3a), and with cortical thickness in their insula and medial prefrontal cortex. In contrast, TSP was negatively correlated with FC between individuals' RVM with their sgACC (RVM-sgACC). When subjects were grouped as pronociceptive or antinociceptive based on whether they had greater thal-BA 3a or RVM-sgACC FC respectively, pronociceptive subjects showed greater TSP responses. Furthermore, TSP was positively correlated with the extent of imbalance toward ascending nociceptive processing. Our study indicates that individuals with enhanced TSP have facilitated ascending nociceptive processing and reduced pain-modulatory capacities.
\end{abstract}

Key words: antinociception; functional connectivity; nociception; RVM; S1; temporal summation of pain

Significance Statement

This study provides novel evidence that an individual's propensity to experience amplified pain with repeated stimuli [i.e., temporal summation of pain (TSP)] reflects attributes of their "pain connectome," namely stronger ascending nociceptive and weaker descending pain-modulatory components. Understanding the individual neural mechanisms underlying TSP within individuals has implications for developing personalized pain-management strategies for chronic pain.

\section{Introduction}

The perception of a noxious stimulus may differ vastly from one individual to another. This likely reflects individual differences in the balance of nociceptive and pain-modulatory processes,

Received Dec. 10, 2014; revised May 26, 2015; accepted May 29, 2015.

Author contributions: J.C.C. and K.D.D. designed research; N.E. performed research; J.C.C., D.D.D., and A.K. analyzed data; J.C.C., N.E., D.D.D., A.K., and K.D.D. wrote the paper.

This work was funded by a grant from the Canadian Institutes of Health Research (MOP 10626). J.C.C. was a recipient of a Queen Elizabeth II/Purdue Pharma Scholarship in Science and Technology, and N.E. was supported by the Fonds National de la Recherche Luxembourg (PDR-09-023). We thank Dr. Adrian Crawley, Dr. Judith Hunter, Dr. Michael Salter, Kasey Hemington, Ruma Goswami, and Marie Andree Coulombe for helpful insights and technical assistance with data analysis and interpretation, and Eugen Hlasny and Keith Ta for expert technical assistance in MRI acquisition.

The authors declare no competing financial interests. termed the pronociception and antinociception spectrum (Yarnitsky et al., 2014). Pronociceptive individuals have facilitated pain processing and/or reduced descending pain-modulatory capacities, whereas antinociceptive individuals display the reverse phenotype (Yarnitsky et al., 2014). The proficiency of these systems may be related to functional synchrony of these brain networks or structural differences.

To interrogate this concept, we examined resting state functional connectivity (FC) and cortical thickness of brain regions

Correspondence should be addressed to Karen D. Davis, PhD, Toronto Western Hospital, 399 Bathurst Street, Room MP14-306, Toronto, 0N M5T 258, Canada. E-mail: kdavis@uhnres.utoronto.ca.

DOI:10.1523/JNEUROSCI.5039-14.2015

Copyright $\odot 2015$ the authors $\quad 0270-6474 / 15 / 359689-12 \$ 15.00 / 0$ 
implicated in pain perception and its modulation, known as the "pain connectome" (Kucyi and Davis, 2015), in relation to individual differences in temporal summation of pain (TSP). TSP refers to the perception of increasing pain in response to repeated noxious stimuli delivered at a frequency of $\geq 0.33 \mathrm{~Hz}$, and is the perceptual correlate of "wind-up" in the spinal dorsal horn (Mendell, 1966; Price et al., 1977). Previous studies of TSP identified large intersubject variability attributed to individual differences in pain processing and/or modulation (Staud et al., 2014). TSP has been linked to the activation of NMDA receptors in the dorsal horn (Davies and Lodge, 1987), and has been shown to be modulated by endogenous opioids (Guan et al., 2006). It has been suggested that ascending processing of TSP in the cortex is mediated by Brodmann area (BA) 3a, but not BA 3b or 1 of the primary somatosensory cortex (S1; Vierck et al., 2013). However, other brain regions implicated in pain processing, such as the secondary somatosensory cortex (S2), anterior/midcingulate cortex (ACC/MCC), prefrontal cortex (PFC), and insula (Davis and Moayedi, 2013), may also play a role in mediating TSP (Staud et al., 2007).

The core of the descending pain-modulatory system is the pathway from the periaqueductal gray (PAG), to the rostral ventromedial medulla (RVM), to the spinal dorsal horn (Fields et al., 1983). The PAG also receives inputs from the hypothalamus, amygdala, PFC, and rostral ACC, which is involved in mediating top-down analgesia (Rainville, 2002; Ossipov et al., 2010). Therefore, strong functional coupling between the sensory thalamus and BA 3a could mediate enhanced TSP response, and individuals with stronger functional coupling between descending nociceptive areas may be better able to attenuate TSP.

To test these concepts, the study aims were (1) to characterize TSP across individuals and (2) to determine the relationship between individual subjects' TSP and the strength of their ascending nociceptive and descending pain-modulation pathways, as reflected by FC and cortical thickness. Coupling psychophysics and brain imaging, we specifically tested the hypotheses that (1) there is broad intersubject variability in TSP magnitude, (2) TSP is positively correlated with FC and cortical thickness within the ascending nociceptive pathway and negatively correlated with FC in the descending pain modulatory pathway, (3) TSP is greater in pronociceptive individuals than in antinociceptive individuals, and (4) TSP is positively correlated with the difference between FC in the ascending nociceptive and the descending painmodulation pathways.

\section{Materials and Methods}

Subjects. Eighty healthy subjects (40 males, 40 females; 19-36 years old) were recruited and provided informed written consent to procedures approved by the University Health Network research ethics board. Exclusion criteria included a previous diagnosis of a psychiatric disorder, taking medication that could affect cognition or pain perception, recurring pain within the previous 6 months, pain lasting $>3$ months within the previous year, and the standard contraindications for MRI (e.g., metal in the body, claustrophobia, or potential pregnancy). All participants underwent a psychophysical session to determine their mean heat-pain threshold and TSP response. They also underwent an MRI session to acquire brain structural and functional data. Data collection was part of a larger study (Erpelding et al., 2012; Erpelding and Davis, 2013; Wang et al., 2014).

Psychophysical session. Heat-pain threshold and TSP were determined for all subjects based on responses to computer-controlled thermal stimuli, delivered to the subjects' left volar forearm with a $30 \times 30 \mathrm{~mm}$ Peltier thermode (TSA-II NeuroSensory Analyzer, Medoc).

Heat-pain threshold was measured using a standard method of limits protocol that we have used previously (Erpelding et al., 2012; Erpelding and Davis, 2013). Each trial began at a baseline temperature of $32^{\circ} \mathrm{C}$, and ramped upwards at $1.5^{\circ} \mathrm{C} / \mathrm{s}$, until the subject pressed a button to indicate that they felt pain. The temperature at this moment was considered their heat-pain threshold. For each subject, their mean heat-pain threshold was taken as the average of the last three of five trials, the first two trials considered training trials and not used.

To determine TSP, a series of 10 consecutive thermal stimuli was delivered to each subject's left volar forearm, using a target temperature of $48^{\circ} \mathrm{C}$ ramped up at $10^{\circ} \mathrm{C} / \mathrm{s}$ from a baseline temperature of $32^{\circ} \mathrm{C}$. Between stimuli, there was a $2 \mathrm{~s}$ interstimulus interval at $40^{\circ} \mathrm{C}$, during which time subjects provided verbal ratings on a scale from 0 to 100 ( 0 , no pain; 100 , worst pain imaginable). Although we had a short interstimulus interval for subjects to provide pain ratings, this was necessary as otherwise the frequency of noxious stimuli would not have been sufficient to generate TSP. As a result of the short interstimulus interval, however, some subjects missed some pain ratings during the first two series of stimuli, and thus the first two series of stimuli were considered training and not used in the analysis. A third series of stimuli was subsequently used to quantify TSP. The magnitude of the TSP effect for each subject was determined based on the percentage change, as well as the absolute change of the pain rating from the first stimulus to the peak pain rating. Three subjects were excluded from further analysis as their initial pain rating was 0 (total of 77 subjects remaining), signaling no pain. Our previous study using the same dataset (Erpelding and Davis, 2013; Wang et al., 2014) determined TSP as a percentage change from subjects' pain rating from the first to the last stimulus. However, in our assessment for this study, we noted that many subjects did not require 10 stimuli to reach their peak pain rating, but either reached a plateau earlier or subsequently diminished. Therefore, here we determined TSP as the percentage and absolute change between subjects' initial and peak pain ratings, to better capture an individual's capacity for TSP. For subsequent analysis, TSP calculated by percentage change from the initial to the peak pain rating was used (see Results, TSP and heat-pain threshold: individual sensitivity and group characteristics).

Brain imaging. Brain imaging data were acquired using 3T MRI (GE Medical Systems). For each subject, we collected a high-resolution T1weighted anatomical scan ( 180 axial slices; $\mathrm{TR}=7.8 \mathrm{~ms}$; $\mathrm{TE}=3 \mathrm{~ms}$; flip angle, $15^{\circ}$; $256 \times 256$ matrix; $1 \times 1 \times 1 \mathrm{~mm}$ voxels $)$ and a $5 \mathrm{~min}$ $\mathrm{T} 2^{*}$-weighted resting-state functional MRI (fMRI) scan (40 slices; TR = $2000 \mathrm{~ms} ; \mathrm{TE}=30 \mathrm{~ms} ; 64 \times 64$ matrix; $3.125 \times 3.125 \times 4 \mathrm{~mm}$ voxels $)$. During the fMRI, subjects were instructed to rest with their eyes closed without guidance to think about anything in particular. One subject did not undergo resting-state fMRI due to technical issues with the scanner. These methods have been previously described (Erpelding and Davis, 2013).

Preprocessing of $f M R I$ data. The resting-state $\mathrm{fMRI}$ data were preprocessed using methods and tools previously described in detail (Kucyi et al., 2013, 2014). Using FSL v5.0 (Jenkinson et al., 2012), the fMRI Expert Analysis Tool (FEAT) was used on each subject's fMRI image to remove the first four volumes, extract the brain using the Brain Extract Tool (BET), correct for motion using the Functional MRI of the Brain (FMRIB) Linear Image Restoration Tool (FLIRT) with Motion Correction, and perform linear registration to both subject's T1-weighted anatomical image [six degrees of freedom (DOFs)] and standard MNI152 2 mm space (12 DOFs) using FLIRT. Subsequently, CompCor (Behzadi et al., 2007; Chai et al., 2012) was used to remove both physiological and scanner-related noise from fMRI data using the tools Matlab (MathWorks) and fMRISTAT (Worsley et al., 2002) in addition to the FMRIB Software Library (FSL). This involved the segmentation of subjects' T1weighted images by FMRIB's Automated Segmentation Tool (FAST) into gray matter (GM), white matter (WM), and CSF partial volume maps. As these were in anatomical space, each subject's WM and CSF partial volume maps were then linearly registered to their fMRI space followed by thresholding of the maps to maintain the top 198 and $20 \mathrm{~cm}^{3}$ probability of voxels respectively, to prevent removal of signal from GM. These thresholded maps were multiplied by the resting-state data, followed by regression of the top five WM and CSF components obtained from whole-brain principal component analysis (PCA). The six motion parameters were also regressed out of the fMRI data, followed by band- 


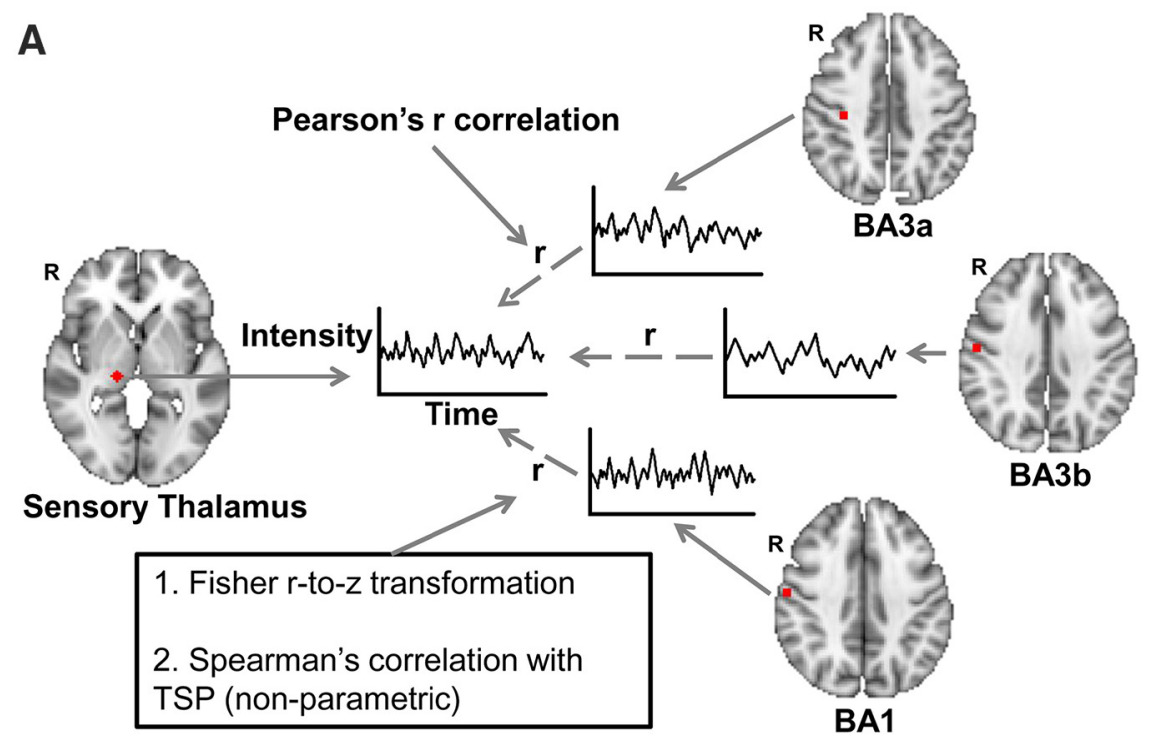

B

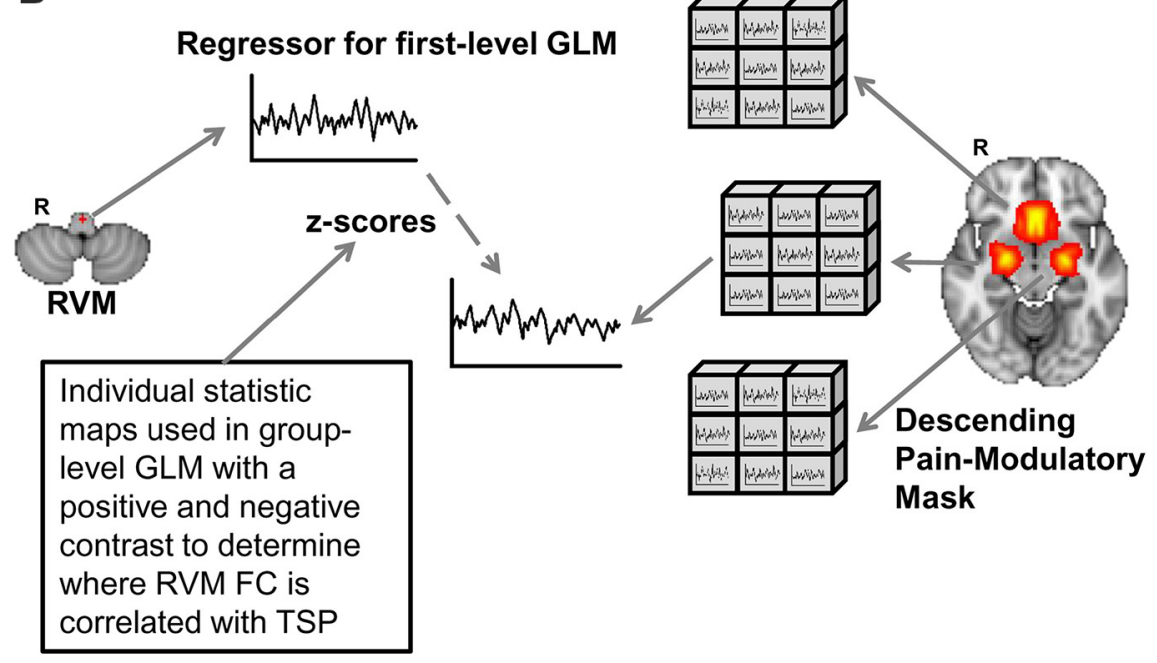

Figure 1. FC analysis in ascending nociceptive and descending pain-modulatory system. $\boldsymbol{A}, 3$-mm-radius spheres were generated in each individual's BA 3a, 3b, and 1, and a 4-mm-radius sphere was generated in each individual's thalamus. For each seed, average resting-state time courses were extracted and each individual's sensory thalamus time course was correlated with the time course of BA 3a, 3b, and 1. The correlation coefficients were then transformed using Fisher $r$-to- $z$ transformations and correlated against TSP. B, A 2-mm-radius sphere was generated in each individual's RVM and average time courses were extracted. A mask of the amygdala and subcallosal cortex was created and each individual's RVM time course was correlated against the time course of each voxel within the mask. Individual z-score maps were used in higher-level GLM with positive and negative contrasts to determine where RVM FC correlated with TSP.

pass temporal filtering $(0.01-0.1 \mathrm{~Hz})$ and spatial smoothing [4 $\mathrm{mm}$ fullwidth at half-maximum (FWHM) kernel].

Ascending nociceptive system seed definitions. As a proxy for the ascending nociceptive system in each subject, we determined the FC between the right sensory thalamus and the right $\mathrm{S} 1$ (BA 3a, 3b, and 1). The right side for the ascending nociceptive system was chosen, as it was contralateral to the stimulation site of the TSP protocol. The FC analysis was determined based on seeds within these areas as follows (Fig. 1A): a 4-mm-radius sphere was generated in the right sensory thalamus (16, $-24,0$ ), which projects to S1 according to the Oxford Thalamic Connectivity Atlas (Behrens et al., 2003a,b). This was specified, as the pathway from the thalamus to BA 3a was hypothesized to be part of a mechanism involved in mediating TSP. Within S1, 3-mm-radius spheres were generated for the right BA $3 \mathrm{a}(34,-22,42)$, BA $3 \mathrm{~b}(54,-12,36)$, and BA 1 $(58,-12,38)$, using the Jülich histological atlas (Eickhoff et al., 2005, 2006, 2007). We used 3-mm-radius spheres in S1 to prevent overlapping of signals between BAs within S1 (compared with a 4 -mm-radius sphere in the thalamus).

Descending pain-modulatory seed definitions. To assess FC of the descending painmodulation system, seeds were placed in two key regions, the PAG and RVM. Specifically, a spherical 6-mm-radius seed was generated in the PAG $(0,-32,-10)$ according to Eippert et al. (2009), and a 2-mm-radius seed in the RVM $(0,-34,-50$; Fig. $1 B)$ based on Vachon-Presseau et al. (2013).

Seed-to-seed FC analysis. As all the seeds were created in standard MNI152 $2 \mathrm{~mm}$ space, they were then linearly registered to each subject's functional space, binarized, and used as masks to extract the appropriate mean intensity time courses from resting-state data. Subsequent $\mathrm{FC}$ analysis was performed by running a Pearson's correlation between the BOLD signal time courses of the right sensory thalamus with the right $\mathrm{BA} 3 \mathrm{a}, 3 \mathrm{~b}$, and 1 in each subject, followed by an $r$-to- $z$ Fisher transformation. The same procedure was repeated between the time course of each subject's PAG and RVM. These Fisher $z$-transformed values were then correlated with subjects' TSP values using a Spearman's $\rho$ correlation. Only FC between each subject's sensory thalamus and BA $3 a$, and FC between the PAG and RVM were hypothesized to be significantly correlated with TSP. Therefore, a Bonferroni's correction for multiple comparisons of these two hypotheses was used, with significance adjusted to $p<0.025$. Although motion correction was performed on resting-state fMRI data during preprocessing, it has been previously demonstrated that subject motion can still cause spurious FC correlations (Power et al., 2012). Therefore, each subject's mean relative displacement between volumes was regressed out of the FC analyses to ensure that the results were not driven by motion. Specifically, the correlation between TSP and subjects' FC between their right sensory thalamus and BA $3 \mathrm{a}, 3 \mathrm{~b}$, and 1 , as well as between the subjects' PAG to the RVM was repeated with a partial Spearman's rank correlation to control for mean relative displacement.

Confirmation of ascending FC results with nonlinear registration. To ensure that the ascending FC results with TSP were specific to $\mathrm{BA} 3 \mathrm{a}$, and not BA $3 \mathrm{~b}$ or 1 , nonlinear registration was used for confirmation because it can provide more accurate registration than linear methods (Klein et al., 2009). Specifically, the sensory thalamus, BA 3a, $\mathrm{BA} 3 \mathrm{~b}$, and BA 1 seeds were nonlinearly registered from standard MNI152 $2 \mathrm{~mm}$ space to each subject's anatomical space using FMRIB's Non-Linear Image Registration Tool (FNIRT; $5 \mathrm{~mm}$ warp resolution; Jenkinson et al., 2012), followed by linear registration (six DOFs) to their functional space. The time courses were then extracted from these masks, and FC analyses were performed as previously stated (see Materials and Methods, Seed-to-seed FC analysis).

Confirmation of descending FC results with brainstem-optimized registration. To confirm the descending-pain modulatory FC results with TSP, the analysis was repeated using automated brainstem coregistration to increase registration accuracy of the brainstem (Napadow et al., 2006). First, linear registration (12 DOFs) from each subject's anatomical space to standard MNI152 $2 \mathrm{~mm}$ space was performed as previously described. Then, a second registration step from anatomical to standard space was 
A

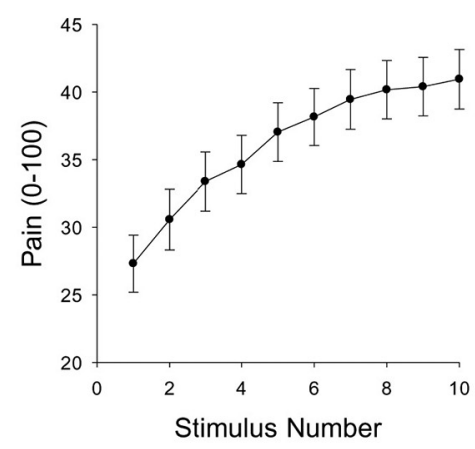

D

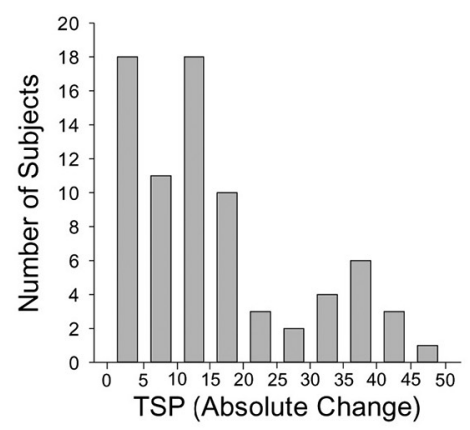

B

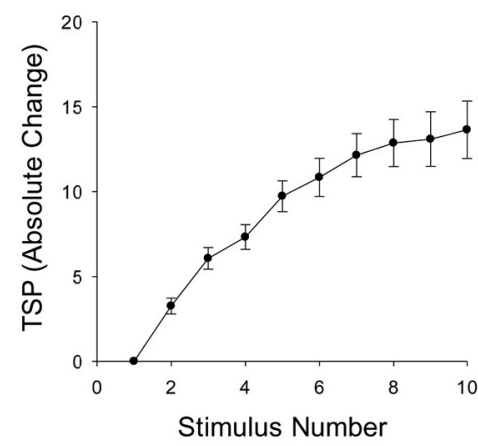

E

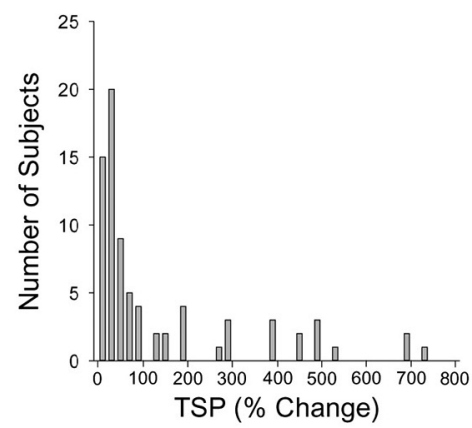

C

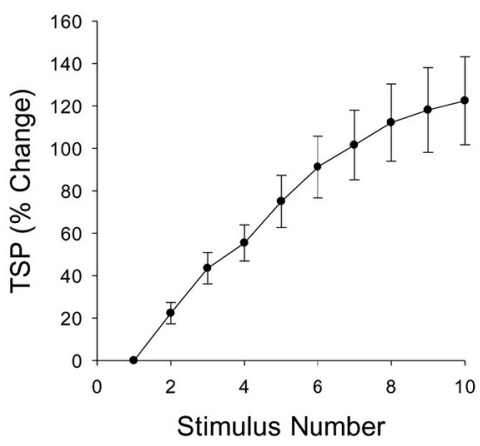

$\mathbf{F}$

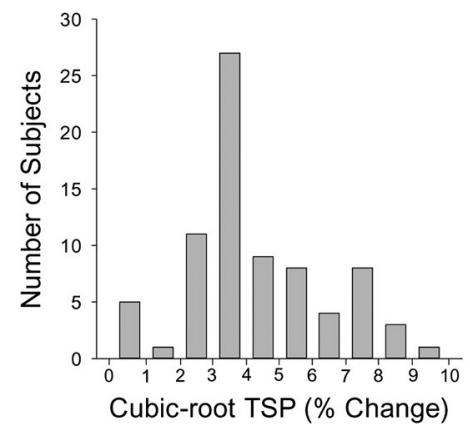

Figure 2. Characterization of TSP. A, Mean group pain rating \pm SE across all subjects for 10 successive stimuli producing TSP. B, C, Mean TSP \pm SE expressed as absolute or percentage change in pain rating from the first to 10 th stimulus across all subjects. $\boldsymbol{D}, \boldsymbol{E}$, Distribution of TSP calculated by absolute or percentage change from subjects' first pain to their peak pain rating. $\boldsymbol{F}$, TSP data transformed into a normal distribution by calculating its cubic root.

performed using linear registration (12 DOFs), with the addition of using a binarized mask of the brainstem created with the Harvard Oxford Subcortical Atlas (Frazier et al., 2005; Desikan et al., 2006; Makris et al., 2006; Goldstein et al., 2007) as a reference volume to weight the correlation ratio cost function in FLIRT. This allows coregistration to be focused on correctly aligning the brainstem (Napadow et al., 2006). The transformation matrix corresponding to each subject's registration from functional to anatomical space created during preprocessing from FEAT was then concatenated with the matrices derived from automated brainstem coregistration, followed by creating the inverse of the concatenated transformation matrix using FLIRT. This inverse matrix was then used to transform the mask of the PAG and RVM created in standard MNI152 2 $\mathrm{mm}$ space to each subject's functional space. Time courses were then extracted from these seeds, and FC analyses were performed as described previously (see Materials and Methods, Seed-to-seed FC analysis).

Seed to descending pain-modulatory mask. No significant correlations were observed between PAG and RVM FC with TSP with either linear registration or automated brainstem coregistration. As an alternative approach to test the relationship between TSP and descending-pain modulatory FC, a mask of regions involved with descending pain modulation was created, which included the bilateral amygdala and subgenual anterior cingulate cortex (sgACC; Eippert et al., 2009). The mask of the amygdala was defined using the Harvard-Oxford subcortical atlas, and the mask of the sgACC was defined using the Harvard-Oxford cortical atlas (Frazier et al., 2005; Desikan et al., 2006; Makris et al., 2006; Goldstein et al., 2007). The regions in the mask were unthresholded and binarized before consequent analysis. The BOLD time course of the RVM in each subject extracted after automated brainstem coregistration was used in a first-level general linear model (GLM) to determine RVM FC with each voxel in the mask after standard linear registration. The differences in registration used for the RVM and for the mask of other brain areas were due to the fact that automated brainstem coregistration increased brainstem registration accuracy to the detriment of the rest of the brain. The statistical maps generated in first-level analyses were then used in a group-level analysis using a mixed-effects (FMRIB's Local Analysis of Mixed Effects (FLAME) 1 and 2) GLM, with demeaned TSP values as a regressor. Both a positive and a negative contrast were used, and significance was determined after mask familywise-error (FWE) correction with a cluster-based threshold of $Z>2.3$ and $p<0.05$. To control for the effects of head motion, this analysis was then repeated with demeaned head-motion values for each subject regressed out of the group-level analysis.

Data transformation. A GLM is a parametric test, and the statistical assumption of normally distributed data was violated because the TSP data were not normally distributed. This does not necessarily mean that the results derived from it are false. Nonetheless, we repeated the seed to descending pain-modulatory mask analysis using demeaned TSP values transformed into a normal distribution as a regressor in GLM during group analysis to verify the previous results. This analysis was also repeated to control for head motion by regressing out demeaned headmotion values for each subject out of the group-level analysis. Because the TSP data was right-skewed (tail on the right), a root transform was used with a cubic-root transformation yielding a normal distribution [one-sample Kolmogorov-Smirnov (K-S) test, $p>0.05$; Fig. $2 F$ ]. A potential concern introduced by root transforms is that it can transform data in different directions because values between 0 and 1 are increased and values $>1$ are reduced by the transformation. However, in this study we did not have TSP values between 0 and 1 , and thus all our data were transformed along the same direction.

In contrast, the correlations between raw TSP with the sensory thalamus FC to the BA 3a, 3b, and 1 were not repeated with cubic-root TSP as a Spearman's correlation was used, which does not assume normally distributed data. Similarly, the correlation between TSP with PAG to RVM FC was not repeated with cubic-root TSP as a Spearman's correlation was used.

Ascending and descending FC balance with TSP. To investigate whether greater FC in the ascending nociceptive processing system is associated with decreased FC in the descending pain-modulatory system, a Pear- 
son's correlation was performed across subjects' FC from their thalamus to their BA 3a (thal-BA 3a), and FC from their RVM to their sgACC (RVM-sgACC). Because a negative trend existed between FC in these two pathways (see Results, TSP is related to the balance between ascending nociceptive and descending pain-modulatory FC), a complementary analysis using stepwise linear regression was performed to confirm their independence. Here, transformed TSP was used as the dependent variable as stepwise linear regression assumes normally distributed variables. The univariate model used descending modulation pathway FC as the independent variable, whereas the bivariate model included the addition of ascending nociceptive pathway FC as the second independent variable.

A group analysis was then performed, where subjects were classified as pronociceptive if they had a greater thal-BA 3a FC compared with RVMsgACC FC and were classified as antinociceptive if they had greater RVM-sgACC FC compared with thal-BA 3a FC. An independent samples $t$ test was then used to determine whether subjects who had a pronociceptive FC (i.e., thal-BA 3a FC greater than RVM-sgACC FC) profile had a significantly greater TSP compared with antinociceptive subjects. To investigate whether the magnitude of TSP was related to the imbalance of ascending nociceptive processing being greater than descending pain modulation, for each subject we subtracted the RVM-sgACC FC from the thal-BA 3a FC. A Spearman's correlation was then determined for this FC difference with TSP across all subjects. The correlation between TSP and FC difference was then repeated while controlling for head motion by using a partial Spearman's rank correlation.

Cortical thickness analysis. The high-resolution anatomical images were for cortical thickness analysis using Freesurfer (http://surfer.nmr. mgh.harvard.edu/), and technical details have been previously described (Fischl and Dale, 2000). Briefly, preprocessing steps included motion correction (Reuter et al., 2010), intensity normalization (Sled et al., 1998), brain extraction, Talairach transformation, tissue segmentation, and formation of surfaces between the WM-GM border and the CSF-GM border. Within each hemisphere, the distance between the two boundaries was then calculated for each point and quantified as cortical thickness.

A GLM was used to test for vertexwise linear correlations of the cortical thickness data, with both TSP and transformed cubic-root TSP within a mask. The mask in the right hemisphere consisted of the S1, S2, insula, ACC, MCC, and medial PFC (mPFC). The regions S1, S2, and mPFC were defined using the PALS-B12 Brodmann atlas (Van Essen, 2005), while the insula, ACC, and MCC were defined using the aparc 2009 atlas (Destrieux et al., 2010). Similar regions were masked in the left hemisphere, with the omission of S1. The total number of vertices for the mask of the right and left hemisphere was 36,085 and 25,337 respectively. Cortical maps were then smoothed with a $6 \mathrm{~mm}$ FWHM kernel, and significance was set at $p<0.025$ (two hemispheres analyzed) following 5000 iterations of Monte Carlo simulation with AlphaSim (http://afni. nimh.nih.gov/afni) to correct for multiple comparisons. Accordingly, 164 and 155 continuous vertices were needed to reach significance in the right and left hemisphere, respectively.

Analysis using pain rating to the first stimulus. The pain rating to the first stimulus was correlated with TSP (see Results, TSP and heat-pain threshold: individual sensitivity and group characteristics). Therefore, we repeated the neuroimaging analyses using the pain rating to the first stimulus as a regressor to determine whether the neuroimaging measures related to TSP were also related to pain to the first stimulus. Within the ascending nociceptive system, a 3-mm-radius sphere in the right BA 3a and a 4 -mm-radius sphere in the right sensory thalamus were linearly registered to each subject's functional space as previously described (see Materials and Methods, Ascending nociceptive system seed definitions; Materials and Methods, Seed-to-seed FC analysis). FC between these two regions was then correlated with pain to the first stimulus across all subjects. The seed to descending pain-modulatory mask FC analysis (see Materials and Methods, Seed to descending pain-modulatory mask) and cortical thickness analysis (see Materials and Methods, Cortical thickness analysis) were also repeated using pain to the first stimulus as a regressor instead of TSP.

\section{Results}

\section{TSP and heat-pain threshold: individual sensitivity and group characteristics}

There were no significant sex differences in mean TSP or heatpain threshold (mean TSP independent samples $t$ test, $p=0.24$; heat-pain threshold independent samples $t$ test, $p=0.37$ respectively), and so data across all subjects were pooled.

At the group level, the 10-stimulus protocol clearly evoked a profile of TSP (Fig. 2). Specifically, pain ratings for each stimulus averaged across all subjects increased with stimulus number (Fig. $2 A)$. Furthermore, the group mean TSP, calculated by either the absolute change or the percentage change from the first stimulus to each successive stimulus, also increased with stimulus number (Fig. $2 B, C$ ). Compared with the first stimulus, mean pain $\pm \mathrm{SE}$ evoked by the 10th stimulus increased by $13.64 \pm 1.69$, corresponding to a percentage change of $122.46 \pm 20.72 \%$. These data also showed marked interindividual differences that are evident by the broad distribution of TSP (Fig. 2D,E). Furthermore, calculating group mean TSP \pm SD as an absolute change from subjects' first stimulus pain to their peak pain rating yielded a change of $17.2 \pm 12.8$. This variability was more pronounced when calculating group mean TSP as a percentage change from subjects' first stimulus pain to their peak pain rating, resulting in a mean TSP $( \pm$ SD) of $140.9 \pm 185.5 \%$.

The greater SE and broader distribution of TSP calculated by percentage changes versus absolute changes reflect greater interindividual variability (Fig. 2, compare $C, B ; E, D$ ), which better serves our study aims to determine brain-behavior associations. Thus, the remaining analyses of TSP were restricted to scores calculated as percentage changes. It is important to note, however, that the TSP responses were not normally distributed (onesample $\mathrm{K}-\mathrm{S}$ test, $p<0.05$ ), and therefore correlations involving TSP used a Spearman's $\rho$, rather than a Pearson's correlation.

Next, we interrogated individual differences in TSP that may be related to an individual's pain sensitivity. We found a positive correlation between subjects' pain rating to the first stimulus and their peak pain rating in the stimulus train (Fig. $3 A$; Pearson's $r=$ $0.777, p=1.00 \times 10^{-13}$ ), but a negative correlation with their TSP (Fig. $3 B$; Spearman's $\rho=-0.725, p=1.91 \times 10^{-13}$ ). Therefore, subjects who perceived greater pain in response to the first stimulus experienced greater peak pain, but a reduced capacity for TSP. In contrast, subjects who required more stimuli to reach their peak pain rating exhibited greater TSP (Fig. 3C; Spearman's $\left.\rho=0.397, p=3.45 \times 10^{-4}\right)$.

The group mean heat-pain threshold \pm SD for all subjects was $45.7 \pm 2.7^{\circ} \mathrm{C}$ and was normally distributed (one-sample K-S test; $p=0.82$ ). There was no significant correlation between subjects' mean heat-pain threshold and their TSP (Fig. 3D; Spearman's $\rho=0.037, p=0.75)$, or the number of stimuli to reach their peak pain rating (Spearman's $\rho=-0.180, p=0.118$ ). However, subjects' heat-pain threshold was negatively correlated with pain to the first stimulus (Fig. 3E; Pearson's $r=-0.26, p=0.02$ ) and with peak pain (Fig. 3F; Pearson's $r=-0.32, p=0.005$ ).

\section{TSP is related to FC of the ascending nociceptive and descending modulatory pathways}

We next examined whether the intersubject variability in TSP was related to the FC of the ascending nociceptive and descending modulation pathways. We found that the magnitude of subjects' TSP was significantly correlated with their FC between the right sensory thalamus and BA 3a (Fig. 4A; Spearman's $\rho=0.269, p=$ 0.019). However, TSP was not significantly correlated with FC between the right sensory thalamus and BA $3 \mathrm{~b}$ or 1 (Fig. $4 A$; 
A
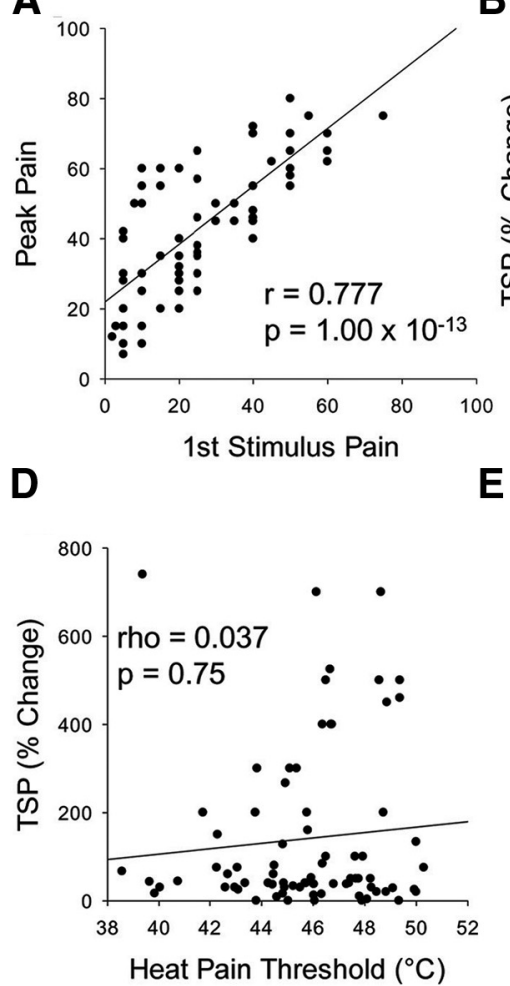

B
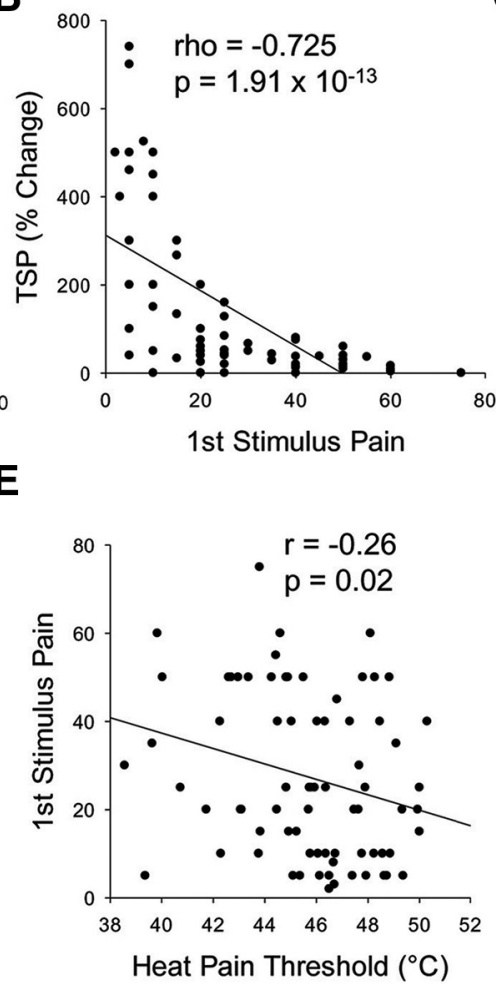

C

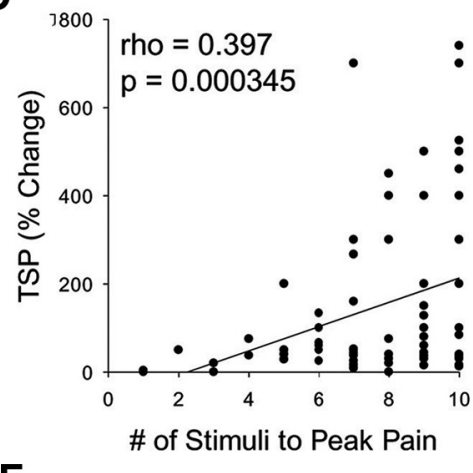

$\mathbf{F}$

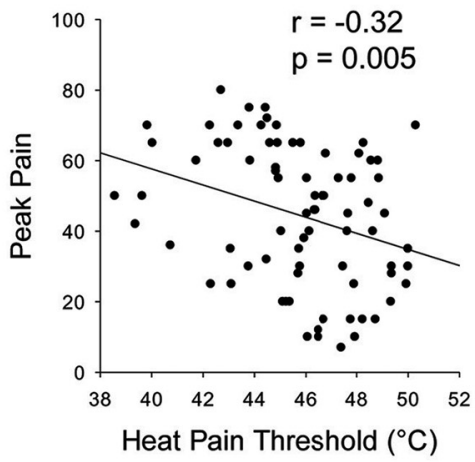

Figure 3. Psychophysical relationships. $\boldsymbol{A}$, Correlation plot of subjects' pain rating to the first stimulus versus their peak pain rating $\left(r=0.777, p=1.00 \times 10^{-13}\right) \cdot \boldsymbol{B}$, Correlation plot of subjects' pain rating to the first stimulus with their TSP response $\left(\rho=-0.725, p=1.91 \times 10^{-13}\right)$.C, Correlation plot of the number of stimuli required for subjects to reach their peak pain rating, with their TSP response $\left(\rho=0.397, p=3.45 \times 10^{-4}\right)$. D. Correlation plot of heat-pain threshold and TSP across all subjects $(\rho=0.04, p=0.75)$. $\boldsymbol{E}$, Correlation plot of subjects' pain rating to the first stimulus with heat-pain threshold $(r=-0.26, p=0.02)$. $\boldsymbol{F}$, Correlation plot of subjects' peak pain rating with heat-pain threshold $(r=-0.32, p=0.005)$.

Spearman's $\rho=-0.031, p=0.793$; Spearman's $\rho=-0.111, p=$ 0.341 , respectively). Repeating the analysis while controlling for head motion (i.e., mean relative displacement) yielded similar relationships between TSP and right sensory thalamus FC with the right BA 3a, 3b, and 1 (BA 3a partial rank Spearman's $\rho=$ $0.285, p=0.013$; BA 3b partial rank Spearman's $\rho=-0.025, p=$ 0.830 ; BA 1 partial rank Spearman's $\rho=-0.108, p=0.359$ ). Verifying the results with nonlinear registration also yielded similar patterns of significance when not controlling for head motion (BA 3a Spearman's $\rho=0.23, p=0.046$; BA 3b Spearman's $\rho=$ $0.004, p=0.973$; BA 1 Spearman's $\rho=-0.115, p=0.323)$, as well as when controlling for head motion (BA 3a partial rank Spearman's $\rho=0.245, p=0.034$; BA 3b partial rank Spearman's $\rho=0.014, p=0.909$; BA 1 partial rank Spearman's $\rho=-0.112$, $p=0.341)$.

Using the seed-based approach, FC between the RVM and PAG was not significantly correlated with TSP when not controlling for head motion (Spearman's $\rho=-0.048, p=0.682$ ), or when head motion was controlled for (partial rank Spearman's $\rho=-0.046, p=0.693$ ). With improved registration using automated brainstem coregistration, the relationship between RVM and PAG FC was still not significantly correlated with TSP when not controlling for head motion (Spearman's $\rho=-0.006, p=$ 0.959 ), or when head motion was controlled for (partial rank Spearman's $\rho=-0.005, p=0.967)$. However, the seed to descending pain-modulatory mask FC analysis revealed a significant negative correlation between TSP and subjects' FC of the RVM with a cluster in the sgACC, based on either original TSP (Fig. 4B; FWE-corrected $Z>2.3, p<0.05$ ) or transformed $\sqrt[3]{\mathrm{TSP}}$ (Fig. $4 B$; FWE-corrected $Z>2.3, p<0.05$ ). When controlling for head motion, the RVM to sgACC FC remained significantly correlated to both original TSP (FWE-corrected $Z>2.3, p<0.05$ ) as well as with transformed $\sqrt[3]{\mathrm{TSP}}$ (FWE-corrected $Z>2.3, p<$ $0.05)$. In contrast, no significant clusters were found with a positive contrast with either original TSP or $\sqrt[3]{\mathrm{TSP}}$ with RVM FC with and without controlling for head motion.

TSP is related to the balance between ascending nociceptive and descending pain-modulatory FC

A correlation of subjects' sensory thalamus to BA 3a FC derived with nonlinear registration with their RVM to sgACC FC yielded no significant relationship not controlling for head motion (Spearman's $\rho=-0.184, p=0.112$ ), as well as when head motion was controlled for (partial rank Spearman's $\rho=-0.186, p=$ 0.110 ). Therefore, having a greater ascending nociceptive FC was not indicative of a lower descending FC. The complementary analysis using stepwise linear regression to verify the independence of the two pathways yielded a significant univariate model $\left(F=19.20, p=3.82 \times 10^{-5} ; \beta_{\text {descending }}=-0.454, p=3.82 \times\right.$ $\left.10^{-5}\right)$. Similarly, the bivariate model including ascending pathway FC as an independent variable was also significant $(F=$ $12.55, p=2.07 \times 10^{-5} ; \beta_{\text {descending }}=-0.424, p=8.65 \times 10^{-5}$; $\beta_{\text {ascending }}=0.225, p=0.03$ ). Importantly, there was an improvement in adjusted $R^{2}$ with the bivariate model $\left(R^{2}=0.235\right)$ over the univariate model $\left(R^{2}=0.195\right)$. Therefore, this analysis reinforces the finding that ascending nociceptive FC and descending pain-modulation FC contribute independently to TSP.

Clustering the subjects by the difference in their ascending nociceptive FC and descending pain-modulatory FC yielded 49 subjects in the pronociceptive group and 27 subjects in the anti- 


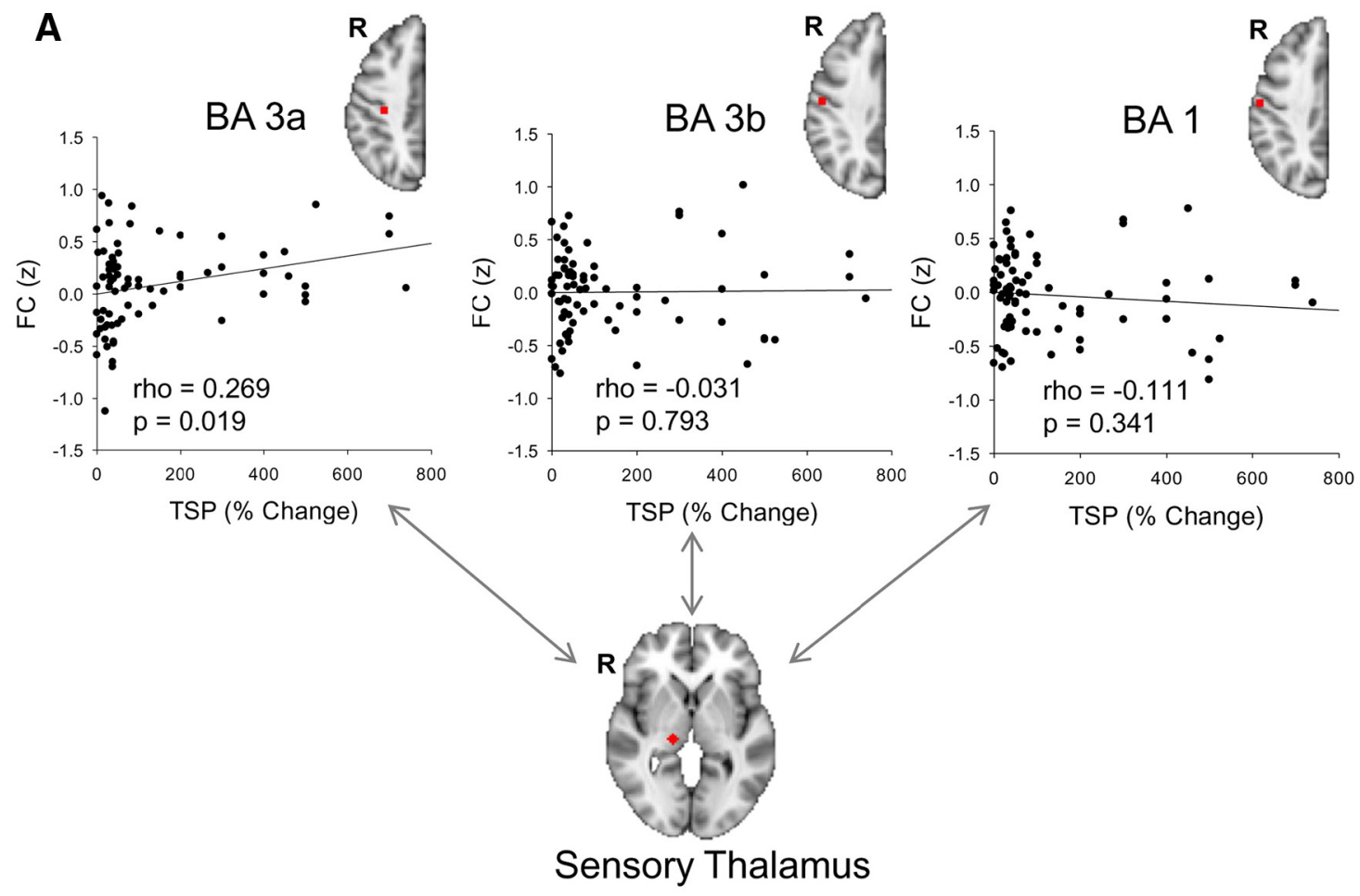

B
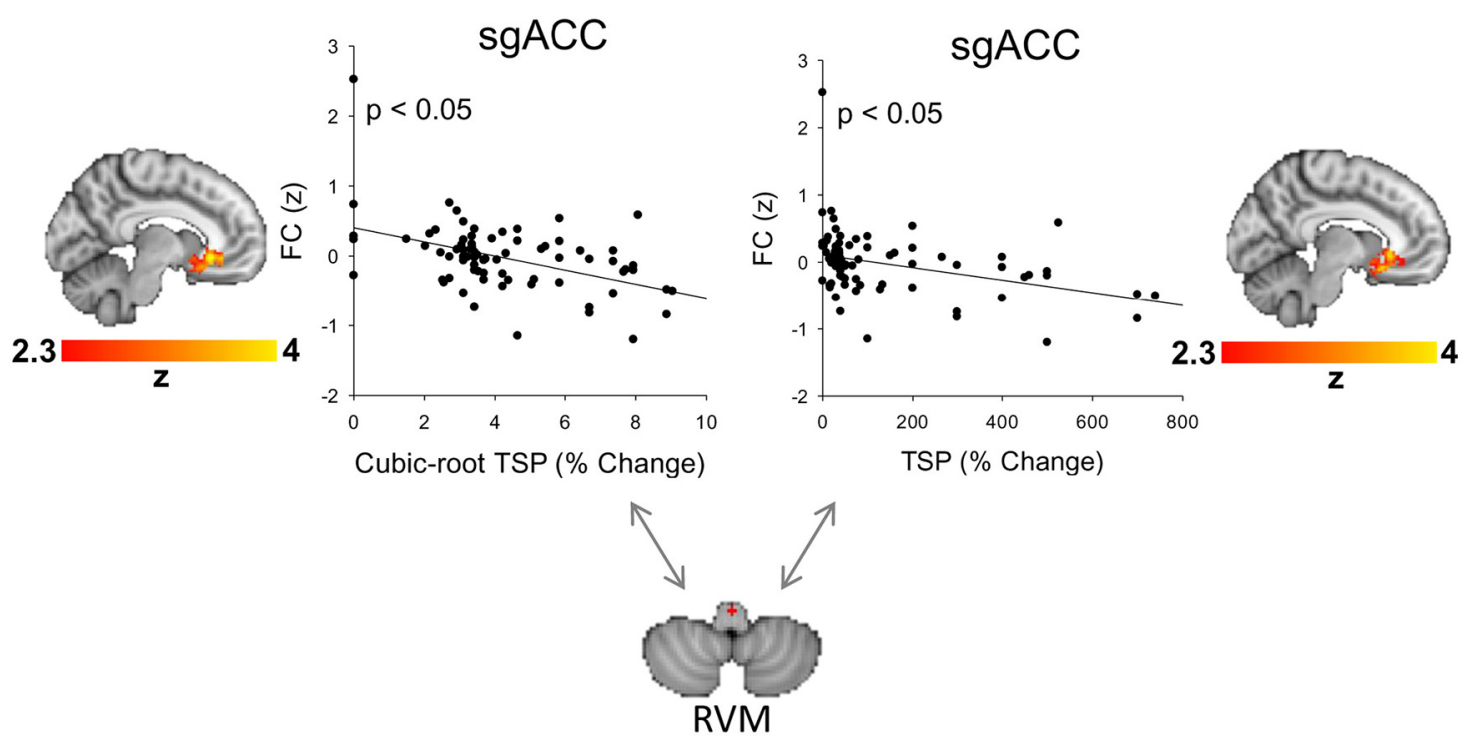

Figure 4. Relationship between $\mathrm{FC}$ of ascending nociceptive and descending pain-modulatory systems with TSP. $A$, Correlation plot of right sensory thalamus $\mathrm{FC}$ to the right $\mathrm{BA} 3 \mathrm{Ba}$, $3 \mathrm{~b}$, and 1 versus $\operatorname{TSP}(\rho=0.269, p=0.019 ; \rho=-0.031, p=0.793 ; \rho=-0.111, p=0.341$, respectively). $\boldsymbol{B}$, Correlation plot of RVM FC to the peak of the sgACC cluster $(-8,28,-8)$ versus transformed TSP (mask FWE-corrected $Z>2.3$, cluster-based $p<0.05$ ) and TSP (mask FWE-corrected $Z>2.3$, cluster-based $p<0.05$ ).

nociceptive group (Fig. 5A). The group mean TSP \pm SD was $185.4 \pm 214.3$ for the pronociceptive group and $63.3 \pm 74.9$ for the antinociceptive group (Fig. 5B). An independent samples $t$ test demonstrated that the pronociceptive group mean TSP was significantly greater than the antinociceptive group mean TSP $(p=0.001)$. Head motion was not significantly correlated with FC difference across all subjects (Spearman's $\rho=-0.155, p=$ 0.181 ), and an independent samples $t$ test showed no significant differences in head motion between pronociceptive and antino- ciceptive subjects $(p=0.053)$. A correlation between TSP and FC difference across all subjects generated a significant positive relationship (Fig. 5C; Spearman's $\rho=0.379, p=0.0007$ ). This relationship was preserved when controlling for head motion (partial rank Spearman's $\rho=0.388, p=0.0006$ ).

\section{TSP and cortical thickness of the pain connectome}

We next examined whether TSP was related to cortical thickness within regions implicated in the pain connectome using a mask 


\section{$\square$ Pro-nociceptive Anti-nociceptive}

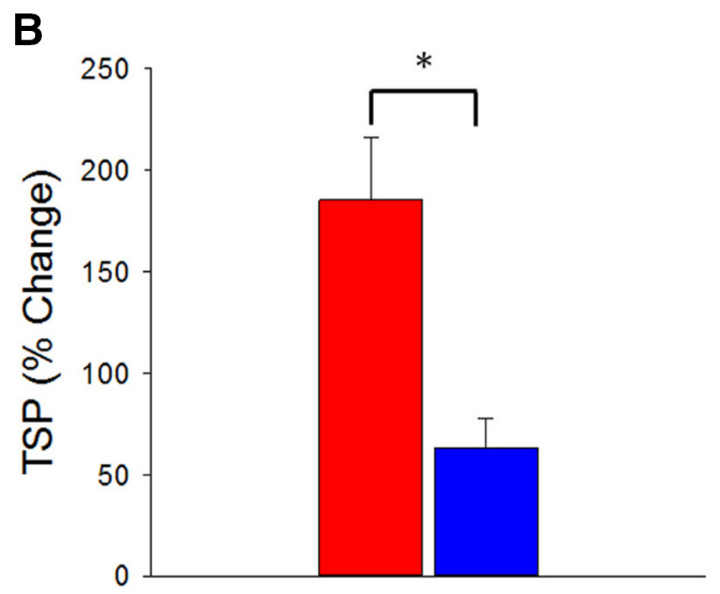

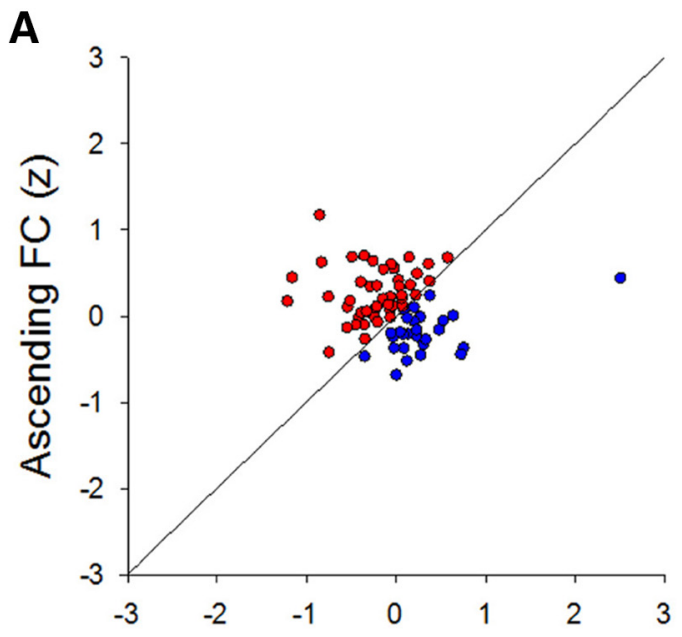

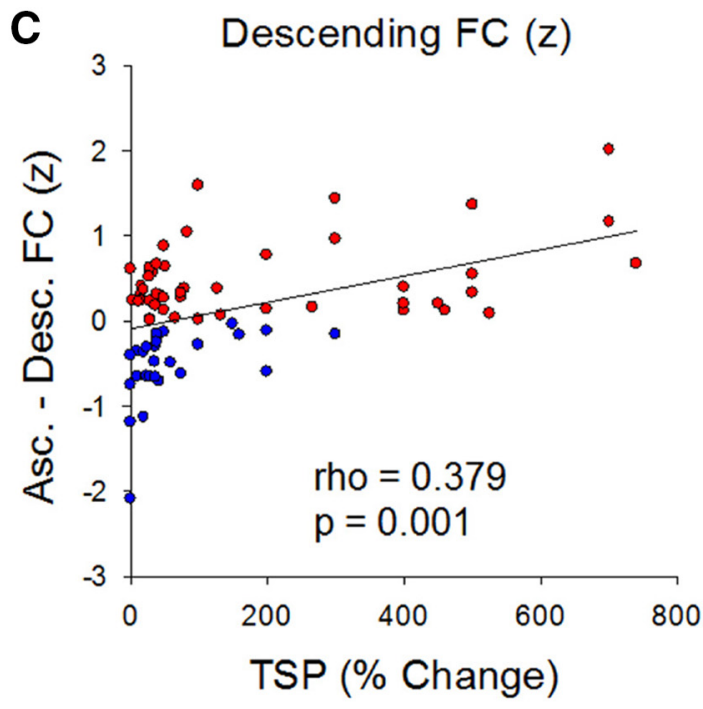

Figure 5. Relationship between TSP and FC difference between ascending nociceptive and descending pain-modulatory pathways. $A$, Plot of subjects' ascending FC versus their descending FC. $B$, Bar graph of group mean TSP \pm SE for pronociceptive and antinociceptive subjects $(p=0.001)$. C, Correlation plot of TSP and FC difference across all subjects (Spearman's $\rho=0.379, p=$ 0.0007). ${ }^{*} p<0.01$.

that included S1, S2, insula, ACC, MCC, and the mPFC. We found that TSP was positively correlated with the cortical thickness in the right insula (Fig. 6A; 268 continuous vertices, Spearman's $\rho=0.435, p=0.001$ ), and in the right mPFC (Fig. $6 B ; 264$ continuous vertices, Spearman's $\rho=0.452, p=0.0012)$. Confirmatory analysis using transformed TSP data $(\sqrt[3]{\mathrm{TSP}})$ yielded similar significant clusters in the right insula (Fig. 6C; 271 continuous vertices, Pearson's $r=0.451, p=0.0008$ ), and in the right $\mathrm{mPFC}$ (Fig. 6D; 222 continuous vertices, Pearson's $r=0.430, p=$ $0.0052)$. In the left hemisphere, there were no statistically significant regions after correction for multiple comparisons.

\section{Pain to the first stimulus}

Pain to the first stimulus was not significantly correlated with FC between the right sensory thalamus and right BA 3a (Pearson's $r=-0.20, p=0.084)$. The seed to descending pain modulatory mask FC analysis as well as the cortical thickness analysis revealed no significant clusters using either a positive or a negative contrast.

\section{Discussion}

This study provides novel evidence that an individual's propensity to experience amplified pain with repeated stimuli (i.e., TSP) reflects attributes of their pain connectome; namely stronger ascending nociceptive and weaker descending pain-modulatory components. Specifically, we showed that (1) there are large interindividual differences in TSP, (2) TSP is unrelated to heat-pain threshold, (3) TSP is positively associated with FC in ascending pain pathways (i.e., FC between the thalamus and BA 3a), (4) TSP is negatively associated with FC in the descending painmodulatory pathway (i.e., FC between the RVM and sgACC), (5) pronociceptive individuals have a greater TSP response than antinociceptive individuals, as TSP is related to the difference in FC between the ascending nociceptive and descending painmodulatory pathways (represented by thal-BA 3a FC minus RVM-sgACC FC), and (6) TSP is correlated with cortical thickness in the right insula and mPFC.

Our behavioral findings support the notion that individuals vary greatly in how pain can become magnified with repeated stimulation. Specifically, we found great intersubject variability in TSP magnitude, as well as the number of stimuli required for a subject to reach their peak pain rating. However, we also observed that the magnitude of TSP is not simply due to pain threshold, as there was no significant correlation between heatpain threshold and TSP. Although both heat-pain threshold and 
A

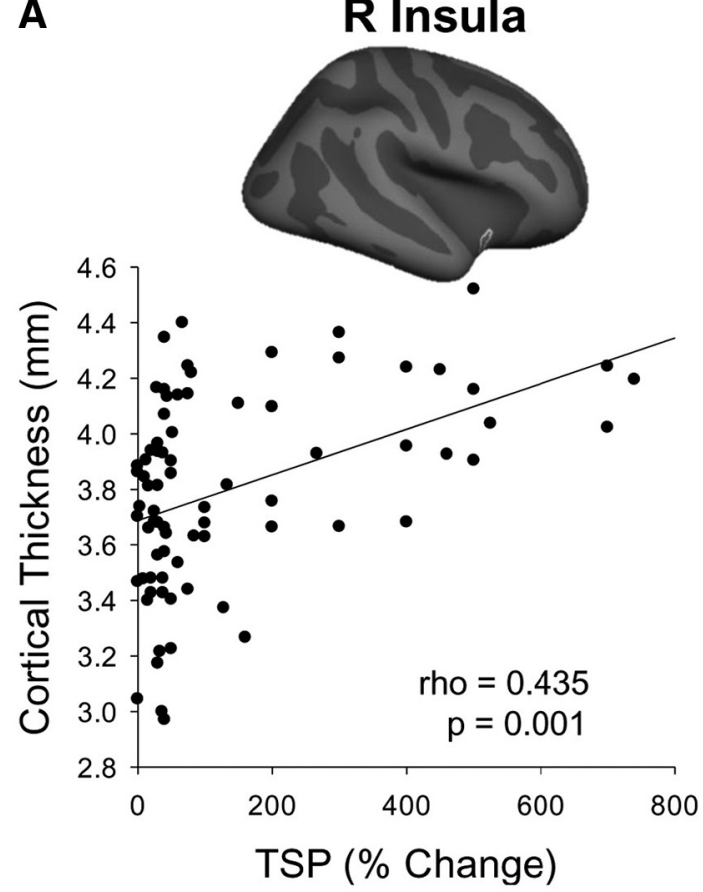

C

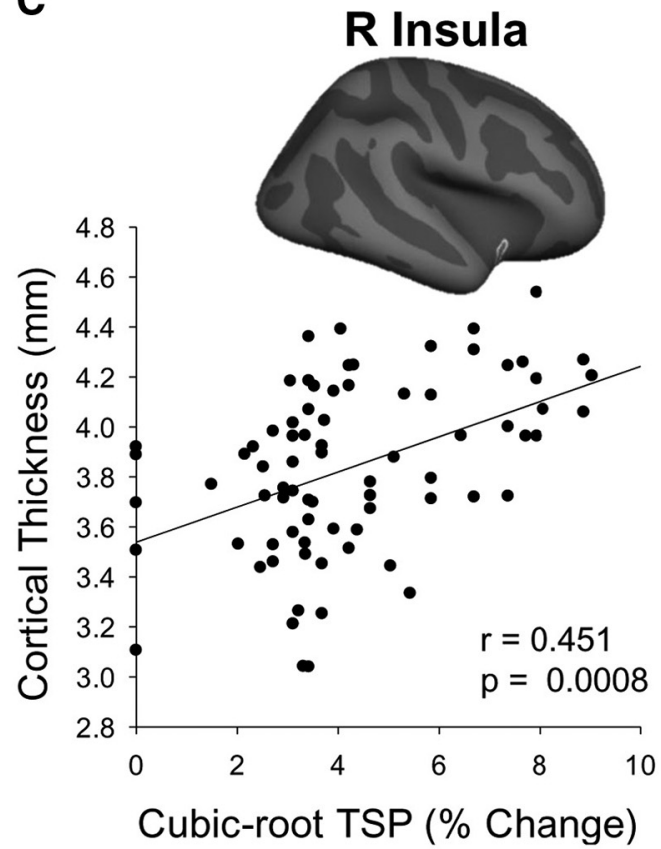

B

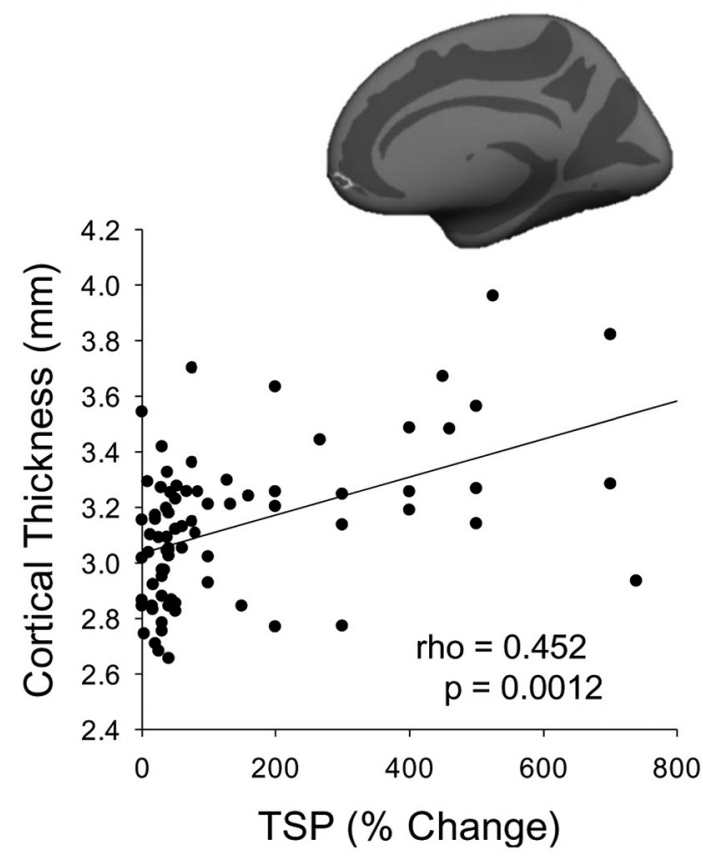

D

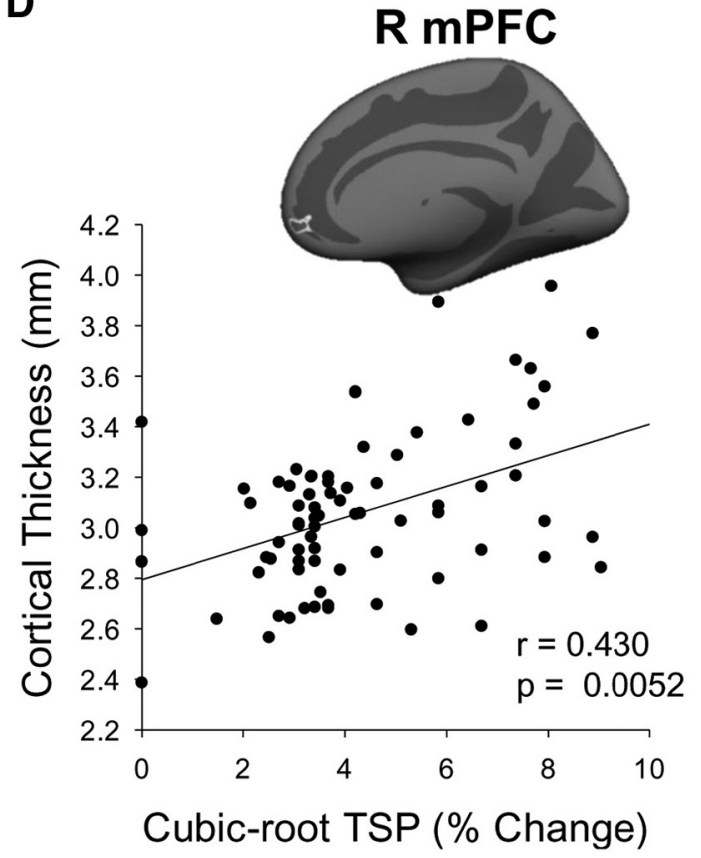

Figure 6. Relationship between cortical thickness and TSP. $\boldsymbol{A}$, Significant positive correlation between insula cortical thickness and TSP ( 268 continuous vertices, $p=0.001)$ across all subjects. $\boldsymbol{B}$, Significant positive correlation between mPFC cortical thickness and TSP ( 264 continuous vertices, $p=0.0012$ ) across all subjects. $\boldsymbol{C}$, Significant positive correlation between insula cortical thickness and $\sqrt[3]{\operatorname{TSP}}(271$ continuous vertices, $p=0.0008)$ across all subjects. $D$, Significant positive correlation between mPFC cortical thickness and $\sqrt[3]{\operatorname{TSP}}(222$ continuous vertices, $p=$ 0.0052 ) across all subjects.

TSP may characterize some aspects of "pain sensitivity," the two measures may be dissociative, and thus mediated by different neural mechanisms. Indeed, different mechanisms have been observed at the level of the spinal cord, where TSP involves activation of NMDA receptors and a wind-up in the dorsal horn (Davies and Lodge, 1987). In contrast, heat-pain threshold is correlated to nociceptive activity, rather than the threshold for action potential firing (Tillman et al., 1995). Psychophysically, we showed a lower heat-pain threshold was associated with a greater pain rating to the first stimulus, as well as a greater peak pain rating in response to the TSP protocol. However, whether the first stimulus and peak pain ratings are greater or lesser, as related to an individual's heat-pain threshold, has no implication on the percentage change between the two values as represented by TSP. For example, an individual with a high heat-pain threshold may rate the first painful stimulus as 10 of 100 , and have a peak pain rating of 20 of 100 , reflecting a $100 \%$ change in TSP, whereas a more sensitive individual with a lower heat-pain threshold may have a first stimulus pain rating of 30 of 100, and a peak pain rating of 60 of 100, also reflecting a 100\% change in TSP. 
Furthermore, our results demonstrate that individuals who have increased spontaneous FC in their ascending nociceptive pathway, and/or decreased FC in their descending painmodulatory pathway have facilitated TSP. Specifically, there was a significant positive correlation between FC of the thalamus to the BA 3 a with TSP, whereas FC to BA $3 \mathrm{~b}$ and 1 did not yield any significant results. This result suggests that $\mathrm{BA} 3 \mathrm{a}$ of the $\mathrm{S} 1$ is part of a mechanism that mediates TSP, in accordance with previous hypotheses (Craig, 2004; Vierck et al., 2013). Additionally, this finding indicates that TSP may be transmitted through peripheral polymodal C-nociceptive fibers that terminate on heat-pinchcold (HPC) cells of lamina 1 of the spinal dorsal horn that project to the posterior part of the ventromedial nucleus of the thalamus (VMpo) and to BA 3 a of the S1 (Vierck et al., 2013). In contrast to the ascending nociceptive pathway, there was a significant negative correlation between FC of subjects' RVM to their sgACC with TSP. Although there are no demonstrated direct projections from the sgACC to the RVM, previous monkey tracer studies have shown connections from the sgACC to the PAG (An et al., 1998; Freedman et al., 2000). In turn, the PAG projects to the RVM to mediate descending pain-modulatory functions, such as the inhibition of nociceptive neurons in the dorsal horn of the spinal cord (Basbaum and Fields, 1978). The ability to detect multisynaptic communications between regions that have no known direct anatomical projections is a benefit of FC analysis, as it offers insight into the underlying functional organization of the brain (Buckner et al., 2013). Therefore, FC between the sgACC and RVM associated with TSP may be routed through the PAG. The lack of a correlation of the RVM FC with the PAG and TSP may be due to confounds, such as the opposing facilitatory and modulatory roles on nociception by the PAG and RVM, as well as the large variety of reciprocal connections between the PAG and other brain regions (Linnman et al., 2012). For example, RVM contains ON cells, which facilitate nociception, as well as OFF cells, which modulate nociception (Fields and Heinricher, 1989). In our study, however, we did not observe a facilitatory relationship between RVM FC and TSP, as no significant clusters were found from a positive contrast.

We did not find a correlation between individuals' FC in their ascending nociceptive pathway (represented by thal-BA 3a FC) and their descending pain-modulatory pathway (represented by RVM-sgACC FC). Therefore, the extent of facilitation in subjects' ascending nociceptive or descending pain-modulatory pathway was not associated with reduction in their descending pain-modulatory or ascending nociceptive capacity, respectively.

The key factor that was found to impact TSP was the balance between the ascending and descending pathways. We classified subjects as (1) pronociceptive if their thal-BA 3a FC was greater than their RVM-sgACC FC, or (2) antinociceptive if their RVMsgACC FC was greater than thal-BA 3a FC. This classification revealed that pronociceptive subjects had significantly greater TSP responses compared with antinociceptive subjects. This significant finding suggests that enhanced TSP is related to an imbalance toward ascending nociceptive processing. Furthermore, TSP was positively correlated with the difference in FC calculated by subtracting each subject's RVM-sgACC FC from their thal-BA 3a FC. Therefore, a greater magnitude of TSP is associated with a greater imbalance toward ascending nociceptive processing and away from descending pain modulation. In contrast, a lesser TSP response is associated with a greater imbalance toward descending pain modulation, and away from ascending nociceptive processing. In terms of the physiological context of FC with pain perception, brain regions that display FC have been shown to coactivate during tasks (Biswal et al., 1995; Smith et al., 2009). Therefore, individuals with enhanced FC in their ascending nociceptive system and/or reduced FC in their descending painmodulatory system may experience greater pain enhancement in response to repeated noxious stimuli, as those networks are more and less coactivated respectively.

We also found significant positive correlations between TSP and cortical thickness of the insula and mPFC. Interestingly, the significant cluster in the insula was located in the anterior portion, an area repeatedly implicated in sustained attention to the salience of pain (Downar et al., 2003; Kucyi et al., 2013), as well as the subjective evaluation of heat pain (Craig, 2009; Wiech et al., 2010). We did not find any association of TSP with cortical thickness in the dorsal posterior insula, which receives projections from VMpo (Craig, 2014), a projection site of HPC lamina 1 spinothalamic tract neurons involved in mediating burning pain (Craig, 2004). One possibility is that although information about TSP is initially routed to the dorsal posterior insula, it is ultimately relayed to the anterior portion of the insula for perceptual decisions on pain (Starr et al., 2009; Wiech et al., 2010). Activity in the mPFC has been implicated in the expectation of pain (Ploghaus et al., 1999), catastrophizing (Gracely et al., 2004), and mind wandering away from pain (Kucyi et al., 2013). Although it was not directly tested here, the significant correlation between mPFC thickness and TSP thus suggests that the MPFC may be involved in the psychological and executive control functions of pain.

Altogether, our work shows that an individual's pain experience may reflect the underlying balance between their ascending nociceptive and descending pain-modulatory pathways. Specifically, an individual's capacity for central sensitization, as reflected perceptually by TSP, and in the spinal dorsal horn as wind-up, is mediated by opposing capabilities for ascending nociceptive processing and descending pain modulation. Accordingly, it has been proposed that individuals who have increased pronociceptive circuit activity, marked by enhanced TSP in the context of our study, may be at a higher risk to develop clinical pain (Yarnitsky et al., 2014). However, individual differences in salience, context, and top-down cognitive factors may also influence individuals' pain experience. Therefore, understanding the complex interplay of psychological factors on fundamental pain processing and pain modulation within individuals, will not only allow a more complete characterization of their nociceptive profile, but will also provide important implications for optimal treatment strategies in chronic pain states. For example, individuals with increased pronociceptive drive (measured through increased TSP) who are predominantly influenced by psychological factors and to a lesser extent by facilitated nociceptive processing and/or reduced pain modulation, may be more responsive to cognitive behavioral therapy, rather than pharmacologic interventions, whereas individuals driven by enhanced pain processing and/or defective pain modulation may benefit more from pharmacological treatments.

\section{References}

An X, Bandler R, Ongür D, Price JL (1998) Prefrontal cortical projections to longitudinal columns in the midbrain periaqueductal gray in macaque monkeys. J Comp Neurol 401:455-479. CrossRef Medline

Basbaum AI, Fields HL (1978) Endogenous pain control mechanisms: review and hypothesis. Ann Neurol 4:451-462. CrossRef Medline

Behrens TE, Johansen-Berg H, Woolrich MW, Smith SM, Wheeler-Kingshott CA, Boulby PA, Barker GJ, Sillery EL, Sheehan K, Ciccarelli O, Thompson AJ, Brady JM, Matthews PM (2003a) Non-invasive mapping of connec- 
tions between human thalamus and cortex using diffusion imaging. Nat Neurosci 6:750-757. CrossRef Medline

Behrens TE, Woolrich MW, Jenkinson M, Johansen-Berg H, Nunes RG, Clare S, Matthews PM, Brady JM, Smith SM (2003b) Characterization and propagation of uncertainty in diffusion-weighted MR imaging. Magn Reson Med 50:1077-1088. CrossRef Medline

Behzadi Y, Restom K, Liau J, Liu TT (2007) A component based noise correction method (CompCor) for BOLD and perfusion based fMRI. Neuroimage 37:90-101. CrossRef Medline

Biswal B, Yetkin FZ, Haughton VM, Hyde JS (1995) Functional connectivity in the motor cortex of resting human brain using echo-planar MRI. Magn Reson Med 34:537-541. CrossRef Medline

Buckner RL, Krienen FM, Yeo BT (2013) Opportunities and limitations of intrinsic functional connectivity MRI. Nat Neurosci 16:832-837. CrossRef Medline

Chai XJ, Castañón AN, Ongür D, Whitfield-Gabrieli S (2012) Anticorrelations in resting state networks without global signal regression. Neuroimage 59:1420-1428. CrossRef Medline

Craig AD (2004) Lamina I, but not lamina V, spinothalamic neurons exhibit responses that correspond with burning pain. J Neurophysiol 92:26042609. CrossRef Medline

Craig AD (2009) How do you feel—now? The anterior insula and human awareness. Nat Rev Neurosci 10:59-70. CrossRef Medline

Craig AD (2014) Topographically organized projection to posterior insular cortex from the posterior portion of the ventral medial nucleus in the long-tailed macaque monkey. J Comp Neurol 522:36-63. CrossRef Medline

Davies SN, Lodge D (1987) Evidence for involvement of N-methylaspartate receptors in 'wind-up' of class 2 neurones in the dorsal horn of the rat. Brain Res 424:402-406. CrossRef Medline

Davis KD, Moayedi M (2013) Central mechanisms of pain revealed through functional and structural MRI. J Neuroimmune Pharmacol 8:518-534. CrossRef Medline

Desikan RS, Ségonne F, Fischl B, Quinn BT, Dickerson BC, Blacker D, Buckner RL, Dale AM, Maguire RP, Hyman BT, Albert MS, Killiany RJ (2006) An automated labeling system for subdividing the human cerebral cortex on MRI scans into gyral based regions of interest. Neuroimage 31:968980. CrossRef Medline

Destrieux C, Fischl B, Dale A, Halgren E (2010) Automatic parcellation of human cortical gyri and sulci using standard anatomical nomenclature. Neuroimage 53:1-15. CrossRef Medline

Downar J, Mikulis DJ, Davis KD (2003) Neural correlates of the prolonged salience of painful stimulation. Neuroimage 20:1540-1551. CrossRef Medline

Eickhoff SB, Stephan KE, Mohlberg H, Grefkes C, Fink GR, Amunts K, Zilles K (2005) A new SPM toolbox for combining probabilistic cytoarchitectonic maps and functional imaging data. Neuroimage 25:1325-1335. CrossRef Medline

Eickhoff SB, Heim S, Zilles K, Amunts K (2006) Testing anatomically specified hypotheses in functional imaging using cytoarchitectonic maps. Neuroimage 32:570-582. CrossRef Medline

Eickhoff SB, Paus T, Caspers S, Grosbras MH, Evans AC, Zilles K, Amunts K (2007) Assignment of functional activations to probabilistic cytoarchitectonic areas revisited. Neuroimage 36:511-521. CrossRef Medline

Eippert F, Bingel U, Schoell ED, Yacubian J, Klinger R, Lorenz J, Büchel C (2009) Activation of the opioidergic descending pain control system underlies placebo analgesia. Neuron 63:533-543. CrossRef Medline

Erpelding N, Davis KD (2013) Neural underpinnings of behavioural strategies that prioritize either cognitive task performance or pain. Pain 154: 2060-2071. CrossRef Medline

Erpelding N, Moayedi M, Davis KD (2012) Cortical thickness correlates of pain and temperature sensitivity. Pain 153:1602-1609. CrossRef Medline

Fields HL, Heinricher MM (1989) Brainstem modulation of nociceptordriven withdrawal reflexes. Ann NY Acad Sci 563:34-44. CrossRef Medline

Fields HL, Bry J, Hentall I, Zorman G (1983) The activity of neurons in the rostral medulla of the rat during withdrawal from noxious heat. J Neurosci 3:2545-2552. Medline

Fischl B, Dale AM (2000) Measuring the thickness of the human cerebral cortex from magnetic resonance images. Proc Natl Acad Sci U S A 97: 11050-11055. CrossRef Medline

Frazier JA, Chiu S, Breeze JL, Makris N, Lange N, Kennedy DN, Herbert MR,
Bent EK, Koneru VK, Dieterich ME, Hodge SM, Rauch SL, Grant PE, Cohen BM, Seidman LJ, Caviness VS, Biederman J (2005) Structural brain magnetic resonance imaging of limbic and thalamic volumes in pediatric bipolar disorder. Am J Psychiatry 162:1256-1265. CrossRef Medline

Freedman LJ, Insel TR, Smith Y (2000) Subcortical projections of area 25 (subgenual cortex) of the macaque monkey. J Comp Neurol 421:172-188. CrossRef Medline

Goldstein JM, Seidman LJ, Makris N, Ahern T, O’Brien LM, Caviness VS Jr, Kennedy DN, Faraone SV, Tsuang MT (2007) Hypothalamic abnormalities in schizophrenia: sex effects and genetic vulnerability. Biol Psychiatry 61:935-945. CrossRef Medline

Gracely RH, Geisser ME, Giesecke T, Grant MA, Petzke F, Williams DA, Clauw DJ (2004) Pain catastrophizing and neural responses to pain among persons with fibromyalgia. Brain 127:835-843. CrossRef Medline

Guan Y, Borzan J, Meyer RA, Raja SN (2006) Windup in dorsal horn neurons is modulated by endogenous spinal $\mu$-opioid mechanisms. J Neurosci 26:4298-4307. CrossRef Medline

Jenkinson M, Beckmann CF, Behrens TE, Woolrich MW, Smith SM (2012) FSL. Neuroimage 62:782-790. CrossRef Medline

Klein A, Andersson J, Ardekani BA, Ashburner J, Avants B, Chiang MC, Christensen GE, Collins DL, Gee J, Hellier P, Song JH, Jenkinson M, Lepage C, Rueckert D, Thompson P, Vercauteren T, Woods RP, Mann JJ, Parsey RV (2009) Evaluation of 14 nonlinear deformation algorithms applied to human brain MRI registration. Neuroimage 46:786-802. CrossRef Medline

Kucyi A, Davis KD (2015) The dynamic pain connectome. Trends Neurosci 38:86-95. CrossRef Medline

Kucyi A, Salomons TV, Davis KD (2013) Mind wandering away from pain dynamically engages antinociceptive and default mode brain networks. Proc Natl Acad Sci U S A 110:18692-18697. CrossRef Medline

Kucyi A, Moayedi M, Weissman-Fogel I, Goldberg MB, Freeman BV, Tenenbaum HC, Davis KD (2014) Enhanced medial prefrontal-default mode network functional connectivity in chronic pain and its association with pain rumination. J Neurosci 34:3969-3975. CrossRef Medline

Linnman C, Moulton EA, Barmettler G, Becerra L, Borsook D (2012) Neuroimaging of the periaqueductal gray: state of the field. Neuroimage 60: 505-522. CrossRef Medline

Makris N, Goldstein JM, Kennedy D, Hodge SM, Caviness VS, Faraone SV, Tsuang MT, Seidman LJ (2006) Decreased volume of left and total anterior insular lobule in schizophrenia. Schizophr Res 83:155-171. CrossRef Medline

Mendell LM (1966) Physiological properties of unmyelinated fiber projection to the spinal cord. Exp Neurol 16:316-332. CrossRef Medline

Napadow V, Dhond R, Kennedy D, Hui KK, Makris N (2006) Automated brainstem co-registration (ABC) for MRI. Neuroimage 32:1113-1119. CrossRef Medline

Ossipov MH, Dussor GO, Porreca F (2010) Central modulation of pain. J Clin Invest 120:3779-3787. CrossRef Medline

Ploghaus A, Tracey I, Gati JS, Clare S, Menon RS, Matthews PM, Rawlins JN (1999) Dissociating pain from its anticipation in the human brain. Science 284:1979-1981. CrossRef Medline

Power JD, Barnes KA, Snyder AZ, Schlaggar BL, Petersen SE (2012) Spurious but systematic correlations in functional connectivity MRI networks arise from subject motion. Neuroimage 59:2142-2154. CrossRef Medline

Price DD, Hu JW, Dubner R, Gracely RH (1977) Peripheral suppression of first pain and central summation of second pain evoked by noxious heat pulses. Pain 3:57-68. CrossRef Medline

Rainville P (2002) Brain mechanisms of pain affect and pain modulation. Curr Opin Neurobiol 12:195-204. CrossRef Medline

Reuter M, Rosas HD, Fischl B (2010) Highly accurate inverse consistent registration: a robust approach. Neuroimage 53:1181-1196. CrossRef Medline

Sled JG, Zijdenbos AP, Evans AC (1998) A nonparametric method for automatic correction of intensity nonuniformity in MRI data. IEEE Trans Med Imaging 17:87-97. CrossRef Medline

Smith SM, Fox PT, Miller KL, Glahn DC, Fox PM, Mackay CE, Filippini N, Watkins KE, Toro R, Laird AR, Beckmann CF (2009) Correspondence of the brain's functional architecture during activation and rest. Proc Natl Acad Sci U S A 106:13040-13045. CrossRef Medline

Starr CJ, Sawaki L, Wittenberg GF, Burdette JH, Oshiro Y, Quevedo AS, 
Coghill RC (2009) Roles of the insular cortex in the modulation of pain: insights from brain lesions. J Neurosci 29:2684-2694. CrossRef Medline

Staud R, Craggs JG, Robinson ME, Perlstein WM, Price DD (2007) Brain activity related to temporal summation of C-fiber evoked pain. Pain 129: 130-142. CrossRef Medline

Staud R, Weyl EE, Riley JL 3rd, Fillingim RB (2014) Slow temporal summation of pain for assessment of central pain sensitivity and clinical pain of fibromyalgia patients. PLoS One 9:e89086. CrossRef Medline

Tillman DB, Treede RD, Meyer RA, Campbell JN (1995) Response of C fibre nociceptors in the anaesthetized monkey to heat stimuli: correlation with pain threshold in humans. J Physiol 485:767-774. CrossRef Medline

Vachon-Presseau E, Martel MO, Roy M, Caron E, Albouy G, Marin MF, Plante I, Sullivan MJ, Lupien SJ, Rainville P (2013) Acute stress contributes to individual differences in pain and pain-related brain activity in healthy and chronic pain patients. J Neurosci 33:6826-6833. CrossRef Medline

Van Essen DC (2005) A population-average, landmark- and surface-based
(PALS) atlas of human cerebral cortex. Neuroimage 28:635-662. CrossRef Medline

Vierck CJ, Whitsel BL, Favorov OV, Brown AW, Tommerdahl M (2013) Role of primary somatosensory cortex in the coding of pain. Pain 154: 334-344. CrossRef Medline

Wang G, Erpelding N, Davis KD (2014) Sex differences in connectivity of the subgenual anterior cingulate cortex. Pain 155:755-763. CrossRef Medline

Wiech K, Lin CS, Brodersen KH, Bingel U, Ploner M, Tracey I (2010) Anterior insula integrates information about salience into perceptual decisions about pain. J Neurosci 30:16324-16331. CrossRef Medline

Worsley KJ, Liao CH, Aston J, Petre V, Duncan GH, Morales F, Evans AC (2002) A general statistical analysis for fMRI data. Neuroimage 15:1-15. CrossRef Medline

Yarnitsky D, Granot M, Granovsky Y (2014) Pain modulation profile and pain therapy: between pro- and antinociception. Pain 155:663-665. CrossRef Medline 